dition of which permits of the satisfactory employment of antiseptics. I have operated with success on the ninth day after the occurrence of perforation, and that this success was largely due to the manner in which the general peritoneal cavity was sealed I have no doubt. When there is suspicion that leakage from the lymph-formed cyst has already occurred (and the occurrence of tenesmus may aid in indicating this) immediate operation is a necessity. As to the manner in which this should be carried out, the condition with which we are dealing should determine.

Early operation, whether demanded by intra-peritoneal rupture, by slower extension, or by leakage from the abscess sac, can best be managed by a median incision supplemented by one in the iliac region. By this means irrigation of the peritoneum can be thoroughly carried out, and the local condition satisfactorily dealt with. When it is determined to operate before the formation of strong adhesions, say within the first five days, I believe that direct incision over the tumour, with careful cleansing and separation of the adherent intestines (for pus may lie in their folds), the examination of the creum and the appendix, and the thorough cleansing of the parts, followed, if necessary, by free drainage, will be very satisfactory. When abscess formation has fully declared itself, I have found that a free incision made as for ligature of the common iliac artery, and long enough to admit of an examination of the parts concerned, followed by antiseptic irrigation and drainage, give satisfactory results. How valuable early operative treatment in one form or other is, the postmortem records of many cases of pyæmia clearly establish. Free incision may not only save a life which is in imminent jeopardy at the time at which it is made, but in it alone lies escape from dangerous sequela. Surgery has in late years done much to add to the measure of human life, and it has been enabled to do this largely through increase in our diagnnstic skill. When this is so developed that perforative appendicitis can be at once recognised, then the quickly widening field will grow broader, and one other condition, which in the past has too often had the saddest issues, will in future by its records serve to point another lesson as to the increasing value of our art.

Carlisle.

ON

\section{SIX CASES OF SUPRAPUBIC LITHOTOMY}

\section{BY ARTHUR NEVE, F.R.C.S. \&c.,} SENIOR SURGEON TO C.M.S. MISSION HOSPITAL, KASHMIR.

THE position and value of suprapubic lithotomy is still sub judice, and will probable remain so for several years. We can hardly estimate either from the small contributions supplied by numerous operators. When surgeons shall be able to report their suprapubic lithotomies by the score, and contrast them with an equally wide experience of other methods treated under the same conditions, then we shall have advanced within measurable distance of judging the operation apart from personal bias and other misleading elements. In the meantime, it is a duty to report the few cases that one has had. From the first I took a keen interest in the subject, and practised Petersen's modification early in 1884, when, as far as I know, no English case had been reported. In the autumin of that year I published an article on the subject in the Indian Medical Gazette. I have up to date had six cases, varying considerably in their nature; of these one ended fatally. They were as follows.

CASE 1 (1884).-R- $\mathrm{R}$, aged forty. A small uric acid calculus diagnosed. As the lithotrite was being repaired litho tomy had to be performed. I injected twelve ounces into the bladder, stuffed the rectum with a large plug of lint, and dissected down on to the anterior surface of the bladder, incised it, and removed a calculus of the size of a large filbert, weight $1 \mathrm{dr}$. $20 \mathrm{gr}$. I stitched the bladder walls at two points, and also closed the abdominal wound. A few drops of urine came by the wound for a day or so, and then stopped; a small quantity of pus collected behind the wound, then escaped, and a few drops were pressed out daily; at the end of three weeks this stopped, but the sinus did not close till the end of a month. Stay in hospital forty-three days.

CASE 2 (1885).-A-, aged twenty-five. The bladder was injected with twelve ounces and the rectal bag was equally distended. After incising the skin, I inserted a curved, sharp-pointed bistoury about one inch and a half above the pubes, and, entering the bladder, cut down to that bone, inserting my finger and forceps as I withdrew the knife, and removed a uric calculus weighing loz. Idr. Thus performed, the operation itself was as prompt as the perineal method; the peritoneum was not seen; and no bleeding ensued.' I put three deep stitches through the bladder and muscles, leaving the skin open. The man suffered for two days from violent, even dangerous, hiccough; but the wound progressed very favourably; in a week it was merely a fine sinus through which urine escaped when he micturated. This closed in about a fortnight more. The patient was thirty-four days in the wards, but for the last three weeks was going about in perfect health.

CASE 3 (1886). - S - aged about twenty-five. The preliminaries were as above, except that only nine ounces were injected into the rectal bag. I dissected down to the bladder in the usual manner. The peritoneum was not seen. After removing the calculus, an oxalic acid one, weighing $13 \frac{1}{2} \mathrm{dr}$. and the size of a bantam's egg, I made a boutonnierre incision in the perineum and inserted a tube in the bladder; then stitched the bladder walls. In spite of the tube and the stitches, most of the urine came by the abdominal wound. For two days the patient had fever ranging from $101^{\circ}$ to $103^{\circ}$. The opening, however, closed very rapidly, and on the twenty-first day was quite closed, and the patient went home. The boutonnidre incision, being useless, was allowed to heal on the fourth or fifth day.

C-1SE 4 (1887). - S-, aged forty-five. From this patient in 1883 I removed a $7 \mathrm{oz}$. calculus, measuring $9 \mathrm{in}$. in circumference, by a double lateral perineal incision. He returned on March 23rd, 1887. The whole bladder seemed full of stone and grit. With some difficulty a few ounces. of fluid were injected, and the rectum distended. In dissecting down, the peritoneum was seen and drawn aside. With much difficulty, on account of adhesion to the bladder walls, a large calculus 4 in. long by 3 in. thick, and irregularly pear shaped, was removed. The bladder walls had to be scraped, but the grit could not be removed. With Dr. E. F. Neve's help I stitched the bladder margins to the muscles, partly closed the wound, and inserted a large drainage tube. The fragments weighed $4 \frac{1}{2} \mathrm{oz}$. In three days he began to get better, and the urine became less offensively ammoniacal. He remained seven weeks in the hospital, and improved in every way. The wound entirely closed, and the urine became more normal. He was treated for some time with one drachm of boracic acid a day in a quantity of water. This set up very great pain in the kidneys, and had to be stopped. The wound, except a small sinus, healed in less than ten days; slight superficial ulceration afterwards. occurred.

CASE 5.-Atma $R$ - - aged forty. This was a stout, well-to-do Hindu. The stone was not large, but was not freely movable in the bladder; this prevented lithotrity being performed. The bladder was injected, and the rectal bag dilated as usual. There was nothing unusual about the operation. The stone ( $1 \frac{1}{2}$ in. by $1 \mathrm{in}$.) was fixed in a sulcus. but was easily removed. The bladder incision was closed by stitches, and the upper margin of the abdominal wound also. A large tube was inserted. For several days the patient did very well. He then had fever, apparently malarial ; and the abundant fat about the wound began to slough. Fourteen days after the operation some of the urine began to come per urethram. The patient then had diarrhoa, which his friends concealed from the hospital. attendants. He daily got weaker, and suddenly the whole subcutaneous tissue of the penis became infiltrated with urine, and sloughed. He was removed by his friends, and died a few days later, about three weeks after the operation.

CASE 6. - M - aged twenty-five, has suffered from symptoms of stone for seven or eight years, and has repeatedly been to hospitals in the Punjab, but at none was any calculus discovered. On sounding, a stone of large size was struck. The operation was performed, with my assistance, by my house surgeon. The bladder and rectum being distended, a dissection was made above the pubes: during its progress the fastening on the penis loosened, and, unperceived, the fluid escaped, leaving the bladder nearly empty. Thus the peritoneum came in sight-occupying, indeed, the whole wound ; it was on the point of being incised when I discovered the mistake. The bladder being reinjected, we saw the peritoneum gradually drawn upwards 
until it could be hooked on one side, exposing the bladder. A very large incision was required for the passace of the stone, which weighed $4 \mathrm{oz} .5 \mathrm{dr}$., and measured in circumference $7 \frac{1}{2}$ in. by $6 \frac{1}{2}$ in. It was so smooth and hard that the forceps did not grasp it. I remored it with the scoop and by pressure from the rectum. The bladder wound was partially closed, and also the upper part of the abilominal wound. Theoperation was performed on June 17th. Although emaciated to a degree, he daily improved for a fortnight, the urine all flowing by the wound. After July sth he had fever, and ten days later was reduced to death's door, with a huge chasm in the site of the wound, diarrhcea, and daily fever. On the day of my return, after a few days' absence, the diarrhea was arrested, and again slow improvement began. Peptonised foods were given, and quinine and resorcin administered. The wound granulated up, and finally healed by August 6th. It left him, however, very weak, although convalescent.

Remarks. - Few conclusions can be drawn from so few and varied cases. For Cases 4 and 6 the suprapubic method was doubtless the best; there was difficulty in removing the stone, even with free access to it. At the same time, in Case 4 a still larger stone had been previously removed by the perineum, and doubtless this could have been thus dealt with. This was the only case in which the peritoneum was visible in the wound. The hiccough in Case 2 was probably due to over-distension of the bladder or rectum, or both; on subsequent occasions I have not injected more than nine ounces into the rectum. From Cases 5 and 6 it appears that the chief danger is derived from the fatty and fascial structures in front of the bladder. If the patient is very stout or is very weak, these tissues are apt to break down, and even if they do not do so primarily, at any check in the progress of recovery the granulations may begin to give way, and there is danger of sloughing, extravasation of urine, putrid absorption, \&c. This is due simply to the bladder draining through the wound. Can this be prevented? A catheter does not appear to act efficiently. By its syphon action it empties the bladder, but does not again act until the bladder is full-that is to say, till the urine is on a level with the highest point of the catheter and a syphon action is again set up, as in the intermittent springs of Derbyshire. The bladder may, indeed, be drained through a small perineal incision with a tube in the bladder; but the same incision slightly enlarger would suffice for small stones, so why complicate it with a suprapubic wound? For large stones it appears to me that such a combination is advisable. Finally, cannot the bladder wound be primarily closed? In all my cases I have aimed at loing so, but in none have obtained complete union. This is chiefly owing to the difficulty of suturing the flaccid bladder walls through a deep small incision. Yet there is no doubt that it is practicable. In my next case I shall proceed as follows :--After exposing the surface of the distended bladder, before incising it, I shall pass long silk sutures on the Lembert principle; that is, having fixed a line of incision, I shall enter the needle half an inch outside it, bring it out one line outside it, cross over to the other side, leaving a long end and a long loop over the site of the wound, re-enter it one line outside, and bring it out half an inch outside, again learing a long end. The sutures should not include the mucous coat. I think with three stitches to the inch (remembering how the wound contracts) the incision in the bladder ought to be thoroughly closed. Having applied all the stitches, an assistant would draw the central loops aside and hold up the blsdder by them and the ends. The incision would then be made and the stone withdrawn. The stitches would then require careful arranging and ligaturing. The bladder should be distended, to test the security of the suturing. The abdominal wound might then be closed, with a small drainage tube left in, and the catheter be tied in or passed every few hours. However the details may require modifying, I am sure that the principle is sound-to aim at primary union of the vesical wound; and that the sutures can best be applied before the bladder has been incised. If primary union or something akin to it can be attained, then the suprapubic operation will be on a par, to say the least, even with the small median perineal incision; but, unless it occurs, the suprapubic incision is attended by many dangers and by protracted healing, and is not likely to continue in farour with the majority of surgeons, except in the case of quite the largest class of stone.

Kashmir

\section{ON SUPRAPCBIC LITHOTOMI.}

BY F. M. SAYDWITH, M.R.C.S.,

HOYORAIY SLRGLON, KASR-EL-ATN HOSPITAL, CAIRO.

CHILDREx in Egypt become the possessors of calculi in their bladders at a very early age. Lately, for instance, I performed lateral lithotomy on a boy, aged eighteen months, whose calculus weighed 201 grains. The following case is that of a boy whose symptoms commenced at two years of age with itching of the penis and anus.

A- , an Egyptian, aged six, was admitted into the Kasr-el-Aini Hospital from the out-patient room with a history of great pain at night, difficult urination and mucous discharge, but nerer blood from the urethra. His penis was abnormally developed, while that of other boys of his age in the hospital measured only $1:$ in. It appeared that a year previously a native doctor had removed with forceps a small stone from the meatus of the penis.

On May 13th, 1888, I performed suprapubic lithotomy. The boy nearly died under chloroform before the operation was beoun, but afterwards took a mixture of ether and chloroform fairly well. This is an exceptional instance, for Egyptians usually take chloroform without any difficulty. The rectum was distended by an indiarubber ball, which was filled with water by a tube attached to it; the bladder was filled with a solution of boracic acid and the penis ligatured by tubing. An incision about two and a half inches long was made reaching to the symphysis pubis ; the peritoneum was not seen; the bladder, when reached, was fixed with a hook, and the stone, weighing 162 grains, was easily extracted with ordinary dressing forceps. Two wire sutures were used for the upper part of the wound, and no drainage tube or catheter was employed.

May 14th: Vomiting yesterday after chloroform. Is now thirsty. Sucks ice. Tongue white. Lies on his side, and urine dribbles from the wound. Temperature $986^{\circ}$.15th: Constipation. Bronchitic râles at both bases of the lungs. Temperature $102 \cdot 2^{\circ}$; pulse 150 ; respiration 40 . Ordered enema, and ipecacuanha mixture for cough.16th: Tongue white and moist. Cough better. Temperature $1004^{\circ}$ A.M., $101^{\circ}$ P.M.; pulse 120 ; respiration 34 . Wound looks healthy. Never pain or tenderness in abdomen.-17th: Ordered enema. Feels hungry. Temperature $100^{\circ}$ A.M., $101^{\circ}$ P.M.; pulse 144 ; respiration $26 .-18$ th: Bronchitic râles still present. Temperature $101 \cdot 2^{\circ}$ A.M., $101 \cdot 8^{\circ}$ P.M.; pulse 136 ; respiration 28 . -20 th (eighth day): Sutures removed. Upper part of wound united, and lower part healthy. Temperature $99.4^{\circ}$ A.M., and $99.2^{\circ}$ P.M.; pulse 120 ; respiration $32 .-24$ th (twelfth day): A few drops of urine from penis for the first time. Temperature $102^{\circ}$, in consequence of pain in a carious tooth and tonsillitis; pulse 128 ; respiration $32 .-26$ th: Urinates now by penis and wound. Temperature $99^{\circ}$; pulse 98; respiration 32.28th: Urine from penis only. Cotton-wool dressing on wound quite dry since yesterday. Wound quite superficial now.-June lst (twentieth day): Wound healed, and child running about ward. To go home.

The fever and other disquieting symptoms in this case were not in any way the result of the surgical wound, though, perhaps, due to the anæsthetic.

Five other cases of suprapubic lithotomy have been done in this hospital during the last twelve months. Mr. Milton's first ease was that of an old man, from whom a large stone was removed, but tbe operation was immediately followed by complete suppression of urine and death. At the necropsy sixteen calculi were found in the two kidneys, and both ureters were blocked by them. His second case was that of a boy aged four, in whom rectal examination discovered the presence of a soft tumour. On opening the bladder on MIay 4th two small polypi were removed. A month later urine still flowed involuntarily from the penis and the wound, but at the end of two months and a half the boy was discharged cured. The third case operated on by a native colleague was on a middle-aged man on April 28th. The stone weighed $7 \frac{1}{4} \mathrm{oz}$, and the patient was discharged cured on June 2 thth The remaining two cases were operated on by another native surcical colleague, without distension of the rectum. The stones were large, and caused some bruising during extraction. One man had a stone removed by suprapubic operation, and left the hospital with a 